\title{
Correction to "In Vivo-to-in Vitro Extrapolation of Transporter-Mediated Renal Clearance: Relative Expression Factor Versus Relative Activity Factor Approach"
}

\begin{abstract}
In the above article [Kumar AR, Prasad B, Bhatt DK, Mathialagan S, Varma MVS, and Unadkat JD (2021) Drug Metab Dispos, 49: 470-478; DOI: https://doi.org/10.1124/dmd.121.000367], the title was incorrectly edited from the manuscript version. The correct title is "In Vitro-to-in Vivo Extrapolation of Transporter-Mediated Renal Clearance: Relative Expression Factor Versus Relative Activity Factor Approach.”
\end{abstract}

The HTML and PDF versions of the article have been corrected.

The compositor apologizes for this error and any inconvenience it caused.

dx.doi.org/10.1124/dmd.120.090969. 